\title{
Duplicity and its Consequences among Variable Stars in General
}

\author{
G. LARSSON-LEANDER (Lund)
}

Abstract

The consequences of stellar duplicity for variable stars in general are reviewed. Gravitational effects, radiation effects and effects due to gaseous streams and mass transfer are considered. The possibility of tidal interactions, modulating the pulsations of $\beta \mathrm{CMa}$ and $\delta$ Sct variables, is among the topics treated in some detail.

\section{Introduction}

In this review the most obvious photometric consequence of stellar duplicity, viz. the occurrence of eclipses, will be omitted. The eclipses are due to specific orientations of the orbital planes of the binaries and have no connection with physical variability.

Because of the presence of a close companion physical variability may, however, be induced in an otherwise non-variable star, and the behaviour of a physically variable star may be more or less influenced by the duplicity. There are at least three different kinds of effects are to be considered:

1. Effects due to the gravitational field of the component star,

2. Effects due to the radiation flux from the component,

3. Effects due to gaseous streams and mass transfer between the components.

The existence of these effects is well-known from observations of eclipsing binaries, but they are, of course, operative also for the class of close binaries that are non-eclipsing.

\section{Gravitational Effects}

Because of the gravitational interaction the level surfaces of the components of a close binary are to a first approximation ellipsoids. Under the orbital motion such tidally deformed systems exhibit periodic light variations with an amplitude generally smaller than 0.1 mag. These are the so-called ellipsoidal variables.

Considerable deviations from the simple ellipsoidal form occur for the very close binaries. This is especially the case for components filling the critical zero-velocity surface. For centrally condensed stars (the Roche model) a convenient method of calculating the distorsion has been described by KOPAL and KITAMURA (1968).

As shown by VON ZEIPEL (1924), half a century ago, the emergent flux of total radiation from surfaces of stars in radiative equilibrium varies in direct proportion to the local gravity. A modification of this theorem, applicable to stars with convective envelopes, has been derived by LUCY (1967). The local value of the effective temperature, $T_{e}$, varies over the stellar surface as

$$
\mathrm{T}_{\mathrm{e}} \sim \mathrm{g}^{\beta}
$$

where $\mathrm{g}$ is the local gravity. In the convective case $\beta \approx 0.08$, while $\beta=0.25$ in the radiative case. Recent numerical calculations of the gravity darkening have been made by several authors, generally in connection with calculation of other proximity effects.

Complicated tidal phenomena arise if the binary orbit is non-circular and/or the axial rotation of the components is non-syndhronous with the orbital motion. The tides affect the elements of an elliptic orbit and have thus great importance for the evolution of a binary system. We cannot here enter on the mathematical theory of tides, but reference is made especially to series of papers by ZAHN (1966 a, b, c) and by KOPAL (1968 a, b, c, d).

It is obvious that a relatively close companion to a pulsating star may influence the pulsations. For stars in the Cepheid instability strip CHRISTY (1966) has shown that the amplitude, phase and shape of the light and velocity variations are determined by the physical 
conditions in and above the hydrogen and helium ionization zones. A sufficiently close companion will produce tides that affect the physical conditions in the outer layers of the pulsating star. Because of this deformation, the amplitude, phase and shape of a radial pulsation will vary in a zonal way over the stellar surface, and due to the orbital motion the orientation of the tidal bulge will vary relative to the line of sight. Under these circumstances it is to be expected that the primary pulsation will show two modulation cycles during one orbital period. Successive cycles will be identical, if the orbit is circular, but will be rather unequal, if the orbital eccentricity is large.

FITCH (1967) proposed the above mechanism as an explanation of the long-period moduexhibited by several stars of the $\beta$ Canis Majoris (or $\beta$ Cephei) and $\delta$ Scuti classes of variables. In his paper FITCH also suggested the possibility that the BLASHKO effect of the RR Lyrae stars is due to the same mechanism. The stars in question would thus be primary components of binaries having relatively faint secondaries. Let us in some detail review recent evidence in support of this proposal. The three classes, $\beta \mathrm{CMa}, \delta$ Sct, and RR Lyr stars, are discussed separately.

Beta Canis Majoris stars. - To substantive his ideas FITCH (1967) performed periodogram analysis of the radial-velocity variations of $\sigma$ Sco. This $\beta \mathrm{CMa}$ star is known to be a single-line spectroscopic binary with a period of either 33.13 or 34.23 days (STRUVE et al., 1955). The period of the primary pulsation is $P_{1}=0.246844$ day. Using a well-distributed set of observations by LEVÉE (1952), FITCH derived, with the assumed period $P_{0}=33.13$ days, new orbital elements, which very satisfactorily represented the nightly mean radial velocities. In this calculation the eccentricity was found as $e=0.40 \pm 0.02$. The series of individual radial-velocity observations, obtained by LEVEE (1952) and by STRUVE et al. (1955), were then represented by means of Fourier expansions in the orbital period $\mathrm{P}_{0}$. As shown by Fig. 1, the variation in the phase zero-point of the primary pulsation is clearly correlated to the orbital motion, and the variation amounts to about $0.1 \mathrm{P}_{1}$ at the time of periastron passage. The small waves, particularly evident in the calculated amplitude variation, are considered by FITCH to be non-real and merely due to observational scatter. FITCH also calculated the tide-raising potential at the centre of the apparent disk of the primary component. From Fig. 2 it is found that the variation of this potential is closely correlated to the variation in phase zero-point of the primary pulsation.

At the $4^{\text {th }}$ Colloquium on Variable Stars, in Budapest 1968, FITCH (1969 a) gave a preliminary report upon tidal resonances in the pulsation of 16 Lac. An augmented and corrected version was published later (FITCH $1969 \mathrm{~b}$ ), together with an analysis of $\beta$ Cep. 16 Lac was discovered to be a spectroscopic binary with period 12.3106 days by STRUVE and BOBROVNIKOFF (1925), while its $\beta$ CMa nature was noted by WALKER (1951). FITCH (1969 b) used radial-velocity observations from 1951-1954, obtained by STRUVE et al. (1952) and by MCNAMARA (1957), and found from the nightly means an almost circular orbit with period $\mathrm{P}_{0}=12.097$ days. Exactly the same value for the orbital period had previously been derived by McNAMARA (1957). FITCH performed periodogram analyses of the radialvelocity observations and of photometric observations 1950-1952, made by WALKER (1951, 1952 , 1954). These analyses disclosed, beside the primary pulsation (period $P_{1}=0.169168$ day, frequency $f_{1}=5.9113 \mathrm{c} / \mathrm{d}$ ), in both velocity and light two more periodic variations (frequencies $f_{2}=5.8529$ and $f_{3}=5.4998 \mathrm{c} / \mathrm{d}$ ). Terms $f_{1}$ and $f_{2}$ are approximately constant in amplitude and phase, while $f_{3}$ is very weak during the first part of the 1951 observing season but is fairly strong at other times. This suggested to FITCH the possibility of a longperiod interference, arising from a close-frequency doublet. FITCH formulated certain criteria, which all have to be satisfied by physically real pulsation frequencies. Application of the criteria showed that $f_{2}$ is a singlet having no connection with tidal perturbations. The criteria also made possible some improvement of the values of $f_{1}$ and $f_{22}$, which made them agree completely with those found by McNAMARA (1957). For $f_{3}$, however, only the doublet members $f_{3}=5.49990$ and $f_{1}-5 f_{0}=5.49799 \mathrm{c} / \mathrm{d}$ were found to satisfy all the criteria. Here $f_{0}$ designates the frequency of the orbital motion. 


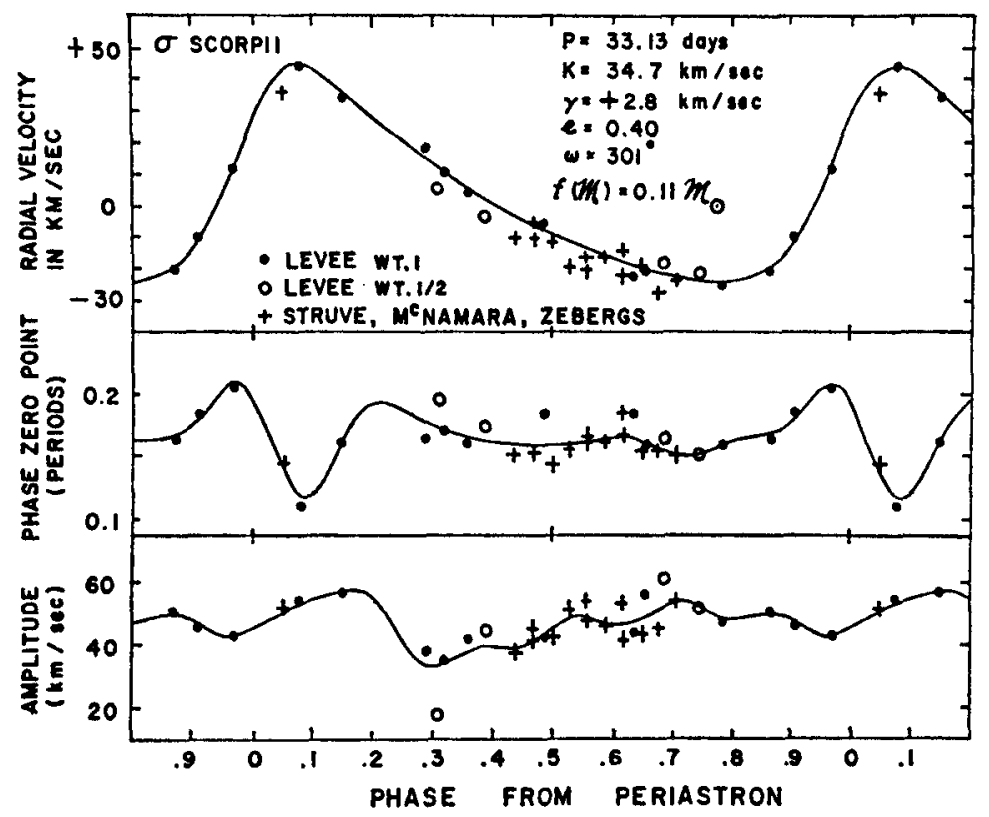

Fig. 1: Analysis of radial velocity variations of $\sigma$ Sco by FITCH. The orbital radial velocity variations of the primary component are shown in the top panel, corresponding variations in phase zero-point and amplitude of the primary pulsation are in the central and bottom panel, respectively. Courtesy The Astrophysical Journal.

The various terms found in 16 Lac have different velocity-to-light amplitude ratios, and only $f_{1}$ can be a radial pulsation-mode. The velocity-to-light amplitude ratio is small for the doublet $\left(f_{3}, f_{1}-5 f_{0}\right)$, and the interpretation given by FITCH $(1969 b)$ is that the star is seen at intermediate inclination, while the doublet members represent equatorial waves. In such a case the light variations may be considerable, although the associated velocity variations are small. The term $f_{3}$ would thus be a non-radial mode, excited by coupling with the primary pulsation through orbital resonance. Term $\mathrm{f}_{2}$ is interpreted as also non-radial, perhaps excited by coupling between the primary pulsation and the rotation of the star.

For $\beta$ Cep FITCH (1969 b) used a large series of radial-velocity observations obtained in 1950-1952 by STRUVE et al. (1953). Periodogram analysis and least-squares fitting gave the frequency of the primary pulsation as $f_{1}=5.2497 \mathrm{c} / \mathrm{d}$. Inspection of the velocity curves suggests a modulation of the amplitude with a period near 11 days. Series of harmonic expansions in frequencies $j f_{1} \pm k f_{0}$, where $j=0,1$ and $k=0,1,2,3,4$, were then tried for various values of $f_{0}$ in a range around the suspected modulation frequency. The residuals from these fits showed at $f_{0}=0.0918 \mathrm{c} / \mathrm{d}$ a sharp minimum in the mean error of a single observation, and this value of $f_{0}$ was adopted as the orbital frequency. With the corresponding period, $P_{0}=10.893$ days, orbital elements were computed, resulting in the eccentricity $e=0.52 \pm 0.15$ and the velocity amplitude $K_{1}=3.1 \pm 0.8 \mathrm{~km} / \mathrm{sec}$. The observed amplitude modulation suggests an intermediate-to-large orbital inclination, and the small value of $K_{1}$ is therefore probably due to a very large mass ratio $M_{1} / M_{2}$. FITCH remarks that if this solution is correct, then minimum pulsation amplitude occurs at or near maximum tidal extension (at periastron passage) and maximum amplitude at maximum compression. 


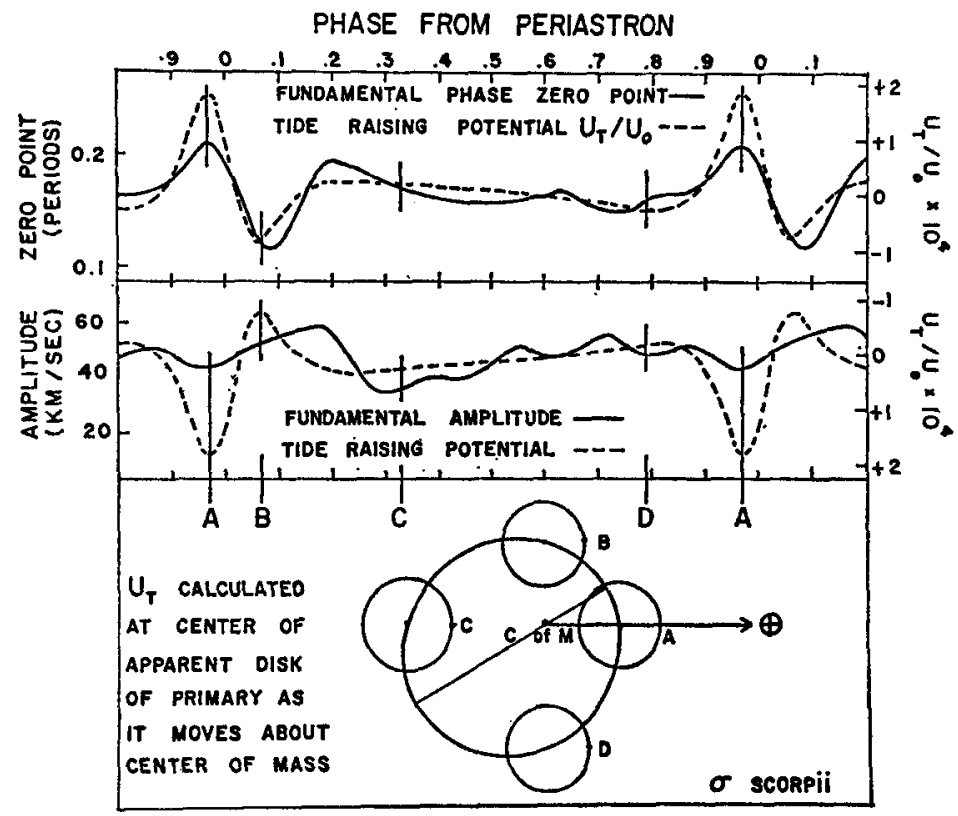

Fig. 2: Analysis of $\sigma$ Sco by FITCH. The variations in phase zero-point and amplitude of the primary pulsation are compared with calculated variation of the tide-raising potential at the centre of the apparent disk of the primary component. Courtesy The Astrophysical Journal.

Other $\beta$ CMa stars showing pulsation modulations that might be due to tidal interaction include $\nu$ Eri and $12 \mathrm{Lac}$ (FITCH, $1969 \mathrm{~b}$ ). The orbital periods for these two stars should then be 15.79 and 8.876 days, respectively.

In his first paper on the tidal effects in pulsating stars FITCH (1967) pointed to the fact that $\beta \mathrm{CMa}$ stars have low rotational velocities, much lower than other stars in the same region of the HR diagram. Observational results indicate that among the $B$ and A stars close binaries generally have lower values of $v \sin i$ than single stars. This has been interpreted as a result of tidal interaction, and the low values of $v \sin i$ for $\beta \mathrm{CMa}$ stars would thus be an additional argument for their binary nature.

In particular FITCH (1967) stated that all known $\beta$ CMa stars have $\mathrm{v} \sin \mathrm{i}<40 \mathrm{~km} / \mathrm{sec}$. Since this paper by FITCH was written the number of $\beta$ CMa stars has increased considerably, due to an extensive photometric search by HILL (1967). He found in the nearest associations and open clusters 24 new variables ascribed to the $\beta$ CMa type. These new variables extend substantially the narrow ranges of spectral type, luminosity class, period, and rotational velocity which have been considered characteristic for the $\beta \mathrm{CMa}$ stars. From tabulations by HILL (1967) and by LEUNG (1967) these ranges, for the 17 previously known, „classical“ variables are, respectively: B $0.5-B$ 3, (II-III)-IV, $3.4-6.9$ hours, and $0-79 \mathrm{~km} / \mathrm{sec}$. The new ranges (HILL, 1967) are: $O 9.5-B 3, I-V, 3-10$ hours, and $0->300 \mathrm{~km} / \mathrm{sec}$. Considering the rotational velocities HILL estimated $v \sin i$ for 19 of the new variables, only for one of these is the limit quoted by FITCH barely satisfied. As pointed out by HILL many of the classical $\beta$ CMa stars were found in radial-velocity surveys, and such surveys generally tend to favour the discovery of variables with sharp lines. Also in other ways the new variables behave differently from the classical ones. Thus, the period-luminosity relation, so evident 
for the previously known variables, is violated by the new ones, as shown by Fig. 3. In an intrinsic-colour-absolute-magnitude diagram, such as Fig. 4 , the new variables populate the same region as numerous non-variable stars. Further observations are needed for these variables, to confirm their nature of bona fide $\beta \mathrm{CMa}$ stars, and for determining more accurate values for the periods.

Finally we should mention here the important discovery, made by SHOBBROOK et al. (1969), that the primary component of the double-line spectroscopic binary $\alpha$ Vir is a $\beta \mathrm{CMa}$ star. The orbital period of the system is 4.014160 days, and most of the observed light variation (yellow amplitude 0.09 mag.) is accounted for by the tidal deformation of the primary. The superposed pulsation has a somewhat variable amplitude, amounting to about 0.032 mag., and a period of 0.173765 day. SMAK (1970) rediscussed all available photometric and radialvelocity data and was able to trace the pulsation also in former velocity measurements. The combined material indicates a decrease of the pulsation period by about 5 seconds per century. According to STRUVE et al. (1958) $a$ Vir A is a fast rotator, with $v \sin i=162 \mathrm{~km} / \mathrm{sec}$. In this respect it is thus reminiscent of some of the $\beta$ CMa stars found by HILL (1967). The system $\alpha$ Vir has further been observed with the Narrabri Observatory intensity interferometer (HERBISON-EVANS et al. 1971). The combination of interferometric data with spectrographic and photometric ones has yielded a wealth of information. In particular the most probable values for the mass and visual absolute magnitude of $\alpha$ Vir $A$ are respectively $10.9 \mathrm{M}_{\odot}$ and -3.5 . The absolute magnitude is in good agreement with the period-luminosity relation for $\beta$ CMa stars as found by LEUNG (1967). It is to be expected that in this close system, with eccentricity 0.146 , tidal modulation of the pulsation will occur and be observable, when a sufficient number of high-quality photometric measurements has been obtained.

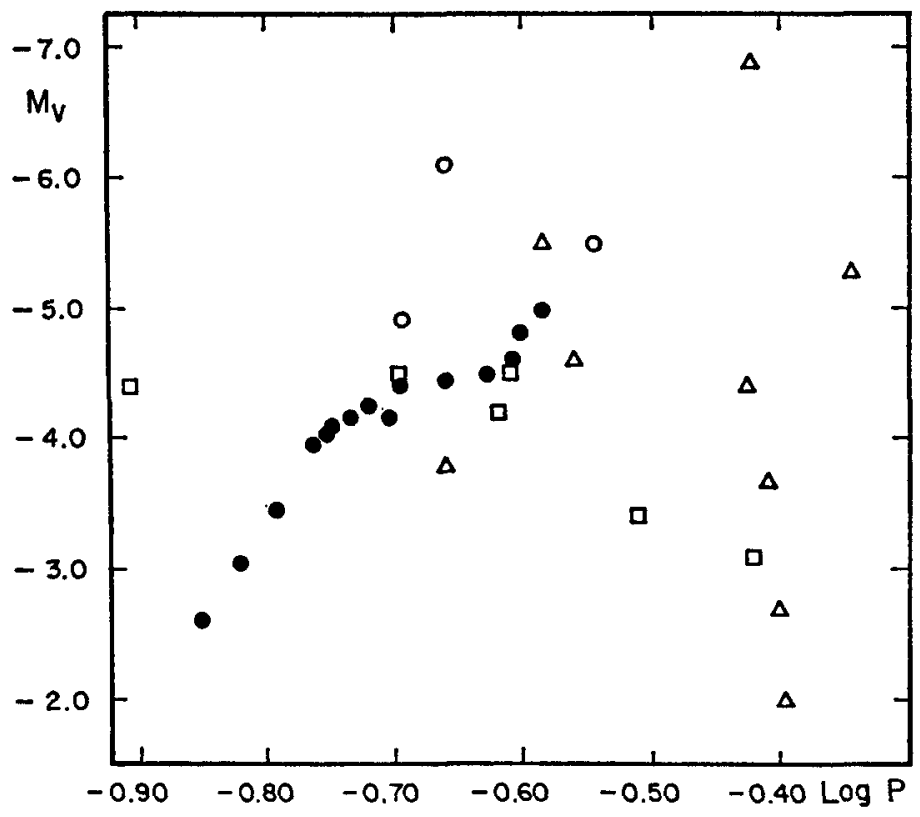

Fig. 3: Period-luminosity relation for $\beta \mathrm{CMa}$ stars. Filled symbols represent the "classical“ variables and open symbols the new ones discovered by HILL. Circles refer to $v \sin \mathrm{i}<100 \mathrm{~km} / \mathrm{sec}$, squares to $100 \leq \mathrm{v} \sin \mathrm{i}<200 \mathrm{~km} / \mathrm{sec}$, and triangles to $v \sin \mathrm{i} \geq 200 \mathrm{~km} / \mathrm{sec}$. Courtesy The Astrophysical Journal. 


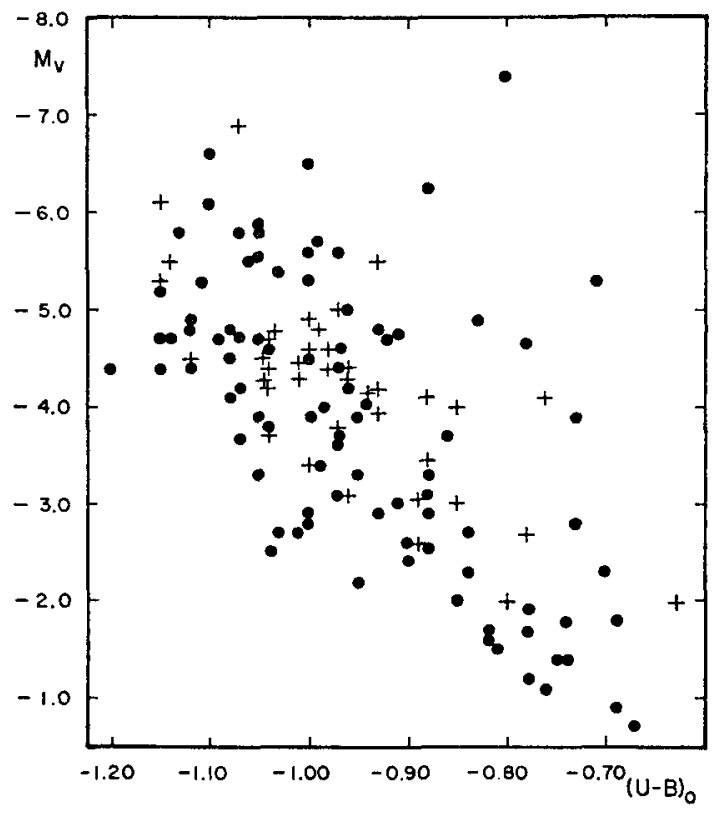

Fig. 4: Intrinsic-colour-magnitude diagram for the stars observed by HILL in his search for new $\beta$ CMa variables. The variables are denoted by crosses and normal stars by circles. Courtesy The Astrophysical Journal.

Delta Scuti stars. - To provide evidence for the suggestion of tidal interaction in the $\delta$ Sct stars, FITCH (1967) made periodogram analysis of the light variations of CC And. The basic observational material is from 1956 and 1957 (FITCH, 1960). The result of the analysis is that the primary pulsation (frequency $\mathrm{f}_{1}=8.0059 \mathrm{c} / \mathrm{d}$ ) is modulated by a period of $\mathrm{P}_{0}=10.466$ days (frequency $\mathrm{f}_{\mathbf{0}}=0.09555 \mathrm{c} / \mathrm{d}$ ). During this long period both amplitude and phase zero-point of the pulsation are exhibiting pairs of distinctly different maxima and minima. The phase variation is accurately repeated over the interval of some $38 \mathrm{P}_{0}$ that is covered by the observations. From this FITCH concludes that the probable explanation is that CC And is a binary with an elliptic orbit and period 10.466 days.

Two of the $\delta$ Sct variables known for some time, viz. $\delta \mathrm{Del}$ and 14 Aur are spectroscopic binaries. $\delta$ Del was reported by PRESTON (1966) as a double-line binary with period 40.5 days and large eccentricity. The light and velocity variations are very complicated, and according to PRESTON the two identical components may both be variable. Suggested pulsation periods are 0.1345 and 0.1568 day. As pointed out by FITCH (1969 b), and provided the interpretation is basically correct, this system offers unique possibilities for studying the influence of gravitational perturbations on the pulsation properties.

The spectroscopic orbit of 14 Aur was derived already by HARPER (1938), the period found is 3.78873 days and the orbital eccentricity 0.033 . From a rather limited number of photometric observations in January 1966 DANZIGER and DICKENS (1967) recognized the star as of the $\delta$ Set type and determined its pulsation period to be 0.122 day. The star has since been the subject of simultaneous photometric and spectrographic observations by CHEVALIER, LE CONTEL, and PERRIN (1968). They found the pulsation period about 0.0938 day (epoch January 1968). Large changes in opposite directions were noted in the amplitudes of the light and velocity curves. As a possible explanation it was suggested that the variable exhibited non-radial pulsations with the pulsation axis along the line joining the centres of the two components. 
Extensive photometric measurements of 14 Aur, mainly by means of the 50 inch automated telescope, were made at Kitt Peak in January 1969 by HUDSON et al. (1971). The light period was determined to 0.08748 day. The secular decrease of the pulsation period, indicated by this value and the previously determined ones, is probably not real, but merely reflecting the increased observational accuracy. HUDSON et al. found that the V amplitude ranged between 0.02 and 0.08 mag. and that the nightly change was progressive, in the sense that minimum magnitude remained almost constant, while maximum magnitude became gradually brighter or fainter. The data were used to test the hypothesis of non-radial pulsations. According to this hypothesis the plot of light amplitude versus orbital phase would show two maxima separated by $180^{\circ}$. There is a peak near phase $340^{\circ}$, and the data are not inconsistent with the presence of a peak near phase $160^{\circ}$, although the distribution of data points is inadequate to prove or disprove the existence of this second peak. Further observations of 14 Aur with the highly efficient automated telescope are most desirable.

During recent years the number of known $\delta$ Sct stars has increased rapidly and now surpasses 40 objects. Sixteen new variables of this type were found in a survey by BREGER (1969a). In addition, FROLOV (1970) has compiled a list of 45 bright stars, which from published photoelectric data are considered as probably $\delta$ Sct variables.

BREGER included his new variables in a general study of the properties of the $\delta$ Sct stars. From this study (BREGER, 1969 b), based on a sample of 39 stars, we extract below some conclusions relevant to the topic of this review.

All the recognized $\delta$ Sct stars belong to luminosity classes $\mathrm{II}-\mathrm{V}$ and are situated in an instability region, between spectral classes $\mathrm{A} 4$ and the border $\mathrm{F} 2 \mathrm{~V}$ to $\mathrm{F} 6$ III. There is a period-luminosity relation existing for the stars, which is approximately an extension of the classical Cepheid relation. An amplitude-luminosity relation exists, stars of high luminosity have larger amplitudes. There is also a relation between $v \sin i$ and amplitude, smaller values of $v \sin i$ correspond to larger amplitudes. For stars of very small amplitude (which are situated. on or near the main sequence) the rotational velocities are similar to stars not recognized as variables, but for stars of larger amplitude the rotational velocities are much lower than for non-variable stars in the same region of the HR-diagram. High values of $v \sin i$ thus seem to prohibit large-amplitude pulsations. From studies of the radial-velocity measurements and from other arguments BREGER concludes that the large majority of $\delta$ Sct stars cannot be close binary systems.

RR Lyrae stars. - As mentioned above FITCH (1967) suggested that the BLASHKO effect could be due to tidal interaction from a faint component. It seems difficult to accept this hypothesis for all RR Lyr stars showing secondary periods, as this phenomenon appears to be very common indeed. In addition the periods change from time to time, whidh also seems difficult to explain on the binary hypothesis. All this, of course, does not preclude the idea that there may be cases of observable tidal interaction among the RR Lyr stars (and among. other stars in the Cepheid instability strip). To the best of my knowledge, however, no direct evidence has been presented as yet.

\section{Radiation Effects}

In the theory of eclipsing binaries the mutual heating of the components is referred to as the reflection effect. To account for the reflection one usually describes the intensity variation of a close binary outside of eclipse by means of an expression

$$
I=A_{0}+A_{1} \cos \Theta+A_{2} \cos ^{2} \Theta
$$

where $\Theta$ is the phase angle and the $A_{i}$ are coefficients to be determined. Tidal distorsion is included approximately as a contribution to the $A_{2}$ term.

When calculating theoretically the reflection contribution to the $A_{i}$ it has been usual to first consider the bolometric radiation and then, assuming the bolometric albedo to be unity, convert to monochromatic radiation by multiplying with a "luminous efficiency factor", whose 
value depends upon an average temperature over the reflecting area. The assumption of unit bolometric albedo is essentially correct when the illuminated stellar atmosphere is in strict radiative equilibrium. In this case the deeper layers are unaffected, and the intrinsic radiative flux of the reflecting component is unchanged. The heated hemisphere radiates the same amount of bolometric flux as it has received from the other component. The assumption fails, however, when the reflecting star has a deep convective envelope (RUCINSKI, 1969). Through the convection the surface heating affects the interior, and the intrinsic flux may be changed. Also the non-illuminated hemisphere will then radiate a flux different from that of the corresponding single star.

A satisfactory geometrical treatment of the photometric reflection effect has been given by NAPIER (1968). In the theoretical computation the binary components are assumed spherical. The illumination of each surface element of a component visible from any point on the other component is considered, except for two small regions in the penumbral zone. Limb darkening is included for both incident and reflected radiation. The conversion to monochromatic light is made without use of a luminous efficiency factor.

NAPIER (1971) applied his method for calculating the theoretical reflection effect for a number of eclipsing binaries previously studied by other authors (HOSOKAWA, 1968; CESTER, 1969), who treated the geometrical problem in a more approximate way. In this rediscussion the most recent scales of temperature and bolometric corrections were used. For binaries with early B-type primaries (having also secondaries of relatively early type) NAPIER found effects in statistical agreement with the observed values. This is taken as an indication that the basic assumptions (local heating and unit bolometric albedo) are approximately correct for the high-temperature stars. In the previous studies of these systems a discrepancy existed between observed and calculated values of the coefficient $A_{1}$, in the sense $O<C$. For the systems having primaries of spectral classes later than $\mathrm{B}_{5}$ (and in most cases sub-giant secondaries) the previous studies gave a large scatter in $\mathrm{O}-\mathrm{C}$ but no systematic discrepancy. In NAPIER's rediscussion of these systems a clear dicrepancy appeared in the sense $\mathrm{O}<\mathrm{C}$. Here the secondaries in most cases have deep convective envelopes, and the basic assumptions, mentioned above, are not fulfilled. A mean albedo of 0.55 was found for these stars, which is in good agreement with the value derived theoretically by RUCINSKI (1969).

To gain a deeper physical understanding of the reflection mechanism and for investigating its monochromatic effects, one must solve the equation governing the transfer of the extraneous radiation through the atmosphere of the reflecting star. Attempts at this were made by SOBIESKI (1965) and by PUSTYLNICK (1967) using theoretical stellar atmospheres, that may be described as slightly non-grey. A step towards a more satisfactory and complete study was taken by RUCINSKI (1970) with an investigation of the Algol system $\mu_{1}$ Sco. The spectral classes of the components are $\mathrm{B} 2 \mathrm{~V}$ and $\mathrm{B} 6.5 \mathrm{~V}$, and radiative equilibrium can thus be assumed. RUCINSKI used the grid of non-grey LTE atmospheres computed by MIHALAS (1965) and applied NAPIER's method for the geometrical treatment of the illumination. The emergent fluxes from the illuminated secondary component were computed for a number of wave-lengths at $\cos \Theta=1.0$ (the sub-stellar point) and at $\cos \Theta=0.4, \Theta$ being the angle between the local normal and the direction to the primary component. The most interesting results are those pertaining to the spectral distribution of the emergent flux from the illuminated component. This distribution was found to be quite different both from that of the non-illuminated hemisphere and from an unilluminated normal atmosphere radiating the same total flux as the illuminated side of the component. In particular, the monochromatic albedo considerably surpasses unity in the Balmer continuum. Thus, close to the Balmer limit the albedo amounts to 1.7 for $\cos \theta=1.0$ and to 2.3 for $\cos \Theta=0.4$. In other spectral regions the deviations from unit albedo are much smaller. RUCINSKI suggests that the large albedo found shortward of the Balmer limit may perhaps explain the ultraviolet excess shown by other Algoltype secondaries (cf. DEVINNEY et al., 1970). To explore this possibility RUCINSKI is planning to make similar computations for models corresponding to later spectral classes than that of the secondary component of $\mu_{1}$ Sco. 
The illumination of the cooler component of a close binary by the hotter one will produce an increase of ionization and excitation that might be observed spectroscopically. This increase should be largest in the sub-stellar region, which is transformed into a kind of "hot spot". It has been speculated (OVENDEN, 1970) that the existence of such a hot spot would induce circulation currents, which might transport hot sub-photospheric gas to the surface. This hypothesis was made in an attempt to explain the presence at all phases of weak highexcitation lines, characteristic for a late O-type star, in the spectrum of the double-line binary $57 \mathrm{Cyg}$ (components of class B $5 \mathrm{~V}$ ). As the cooling time for material in a B-type atmosphere is only of the order of 1 second NAPIER and OVENDEN (1970) abandoned this explanation, and they now favour the idea that the high-excitation lines originate in a chromosphere, formed above each of the two hot spots as a result of turbulence, due to temperature differences over the stellar surfaces.

\section{Gaseous Streams and Mass Transfer}

A comprehensive review of gaseous streams and other manifestations of circumstellar matter in close binary stars has recently been given by BATTEN (1970). It is therefore unnecessary to go into details here, and we merely summarize the main points. We find evidence for circumstellar matter in binary systems from six different kinds of observations.

The most direct evidence is emission features in the spectra. A large proportion of close double stars exhibit emission lines of mainly hydrogen. In many eclipsing binaries, especially of the Algol type, the lines show variations in intensity, profile, and velocity, clearly indicating that they originate from gaseous rings around the primary components. In other systems emission is observed only at certain phases out of eclipse, implying gas streams between the components.

A second direct evidence is the distortion of velocity curves due to the blending of the stellar lines with absorption lines formed in gas moving between and around the components. This distortion manifests itself in a number of ways. One that was early noted is the BARR effect, the statistical preference among close binaries with elliptical orbits for small values of $\omega$, the longitude of periastron.

In order to derive reliable orbital elements one should ideally try to eliminate blending absorption and emission features from the stellar lines through spectrophotometric studies of line profiles at a variety of phases. Conversely, spectrophotometry is also a means to obtain information on the circumstellar matter. It is thought that a deeper insight into the physical state of the gaseous streams and envelopes will emerge as a result of this technique. However, before this goal is reached, many more observations are necessary. The discussion of obtained spectrophotometric data is often complicated, as possible reflection effects and effects due to gravity darkening have also to be considered.

During recent years intrinsic polarization of light has been observed for a number of close binaries, indicating the presence of free electrons in surrounding shells or disk-like structures. This is our fourth type of observational evidence for circumstellar matter.

The light curves of eclipsing binaries exhibit changes and anomalies, which may be caused by varying opacity of the circumstellar matter. This evidence for circumstellar matter is less direct than those mentioned above, and admittedly rather large amounts of matter are necessary to account for changes observed in the continuous radiation. In some cases anomalies of light curves may be due to hot spots, which are different from those originating from reflection. GORBATSKY (1969) argues that these hot spots might be regions of the envelopes around primary components that are shock-heated by a stream of particles travelling from the secondary component.

The sixth evidence for circumstellar matter is found in the period changes that are observed for eclipsing binaries. There are both progressive changes and more abrupt ones. In the absence of a third body the changes are interpreted as effects due to mass loss and mass transfer between components. Isotropic mass loss is always increasing the period, but directional mass loss and mass transfer can change the period in either direction. The dynamical 
effects of mass loss and of mass and angular momentum transfer have been reviewed in detail by HUANG (1966) and by KRUSZEWSKI (1966). As is well known, these effects are very important in the theories of evolution of close binaries. Especially dramatic effects of mass transfer occur in certain very short-period systems, as the transfer seems to trigger the outbursts of novae and U Gem variables. This topic is discussed by Dr. SMAK in a later section of this volume.

Large-scale mass transfer is essential in the theory of $\beta \mathrm{CMa}$ instability proposed by STOTHERS and SIMON (1969). According to these authors pulsational instability of the $\beta$ CMa type is due to an inversion of the mean molecular weight in the outer layers of a star. This $\mu$-mechanism would operate if the $\beta C M$ a variables were mass-accreting components of close binaries and if the mass transfer had proceeded far enough that the mass-losing components, in the post-main-sequence state, had lost not only all of the hydrogen-rich envelopes but also material of higher molecular weight. The theory has been criticized by PLAVEC (1971), just because of the very large mass transfer that is necessary. Further criticism will be given in a subsequent paper by Dr. PERCY.

To end this review I think it is proper to mention the fascinating possibility that collapsars or black holes might eventually be shown to exist from observations of binary systems. In particular, it has been suggested by CAMERON (1971) that the disk-like structure known as the so-called infrared component of $\varepsilon$ Aur may contain a central collapsar. STOTHERS (1971) has pointed out that a similar object with a disk may be in orbit around the super-giant $98 \mathrm{Her}$.

The subject of this review is a vast one with many aspects. Several items have not been covered properly or have not been taken up at all. Among the latter ones are magnetic interaction between components and the possible rôle of duplicity for symbiotic stars, shell stars, Am stars etc. For all omissions and other shortcomings on the part of the reviewer $I$ ask for your indulgence.

$$
\text { References: }
$$

BATTEN, A. H., 1970, Publ. Astron. Soc. Pacific 82, 574.

BREGER, M., 1969a, Astrophys. J. Suppl. 19, 79.

BREGER, M., 1969b, Astroph ys. J. Suppl. 19, 99.

CAMERON, A. G. W., 1971, Nature 229, 178.

CESTER, B., 1969, Mem. Soc. Astronomica Italiana 40, 169.

CHEVALIER, C., Le Contel, J. M., and PERRIN, M. N., 1968, Astrophys. Letters 2, 175.

CHRISTY, R. F., 1966, Astrophys. J. 144, 108.

DANZIGER, I. J., and DICKENS, $\dot{R}$. J, 1967, Astrophys. J. 149, 55.

DEVINNEY, E. J., HALL, D. S, and WARD, D. H., 1970, Publ. Astron. Soc. Pacific 82, 10.

FITCH, W. S., 1960, Astrophys. J. 132, 701.

FITCH, W. S., 1967, Astrophys. J. 148, 481.

FITCH, W. S., 1969a, in L. Detre (ed.) Non-Periodic Phenomena in Variable Stars, D. Reidel Publ. Comp., Dordrecht, p. 287.

FITCH, W. S., 1969b, Astrophys. J. 158, 269.

FROLOV, M. S., 1970, Inform. Bull. Var. Stars, No. 427.

GORBATZKY, V. G., 1969, in M. Hack (ed.) Mass Loss from Stars, D. Reidel Publ. Comp., Dordrecht, p. 271 .

HARPER, W. E., 1938, Publ. Dominion Astrophys. Obs. 6, 12.

HERBISON-EVÁNS, D., BROWN, R. H., DAVIS, J., and ALLEN, L. R., 1971, Monthly Notices Roy. Astron. Soc. 151, 161

HILL, G., 1967, Astrophys. J. Suppl. 14, 263.

HOSOKAWA, Y., 1968, Bull. Yamagata Univ. (Natural Science) 7, 7 (Sendai Rap. No. 101).

HUANG, S.-S., 1966, Ann. Rev. Astron. Astrophys. 4, 35.

HUDSON, K. I., CHIU, H.-Y., MARAN, S. P., STUART, F. E., and VOKAC, P. R., 1971, Astrophys. J. $165,573$.

KOPAL, Ż., 1968a, Astrophys. Space Sci. 1, 179

KOPAL, Z., 1968b, Astrophys. Space Sci. 1, 284.

KOPAL, Z., 1968c, Astrophys. Space Sci. 1, 411.

KOPAL, Z., 1968d, Astrophys. Space Sci. 2, 48.

KOPAL, Z, and KITAMURA, M., 1968, Adv. Astron. Astrophys. 6, 125.

KRUSZEWSKI, A., 1966, Ad'v. Astron. Astrophys. 4, 233.

LEUNG, K.-C., 1967, Astrophys. J. 150, 223.

LEVEE, R. D., 1952, Astrophys. J. 115, 402.

LUCY, L. B., 1967, Z. Astrophys. 65, 89.

MeNAMARA, D. H., 1957, Astrophys. J. 125, 684.

MIHALAS, D., 1965, Astrophys. J. Suppl. 9, 321.

NAPIER, W. McD., 1968, Aistrophys. Space Sci, $2,61$. 
NAPIER, W. McD., 1971, Astrophys. Space Sci. 11, 475.

NAPIER, W. MeD., and OVENDEN, M. W., 1970, Astron. Astrophys, 4, 129.

OVENDEN, M. W., 1970, Vistas in Astron. 12, 135.

PLAVEC, M., 1971, Publ. Astron. Soc. Pacific 83, 144.

PRESTON, G. W., 1966, Kleine Veröff. Remeis-Sternw. Bamberg, No. 40, p. 163.

PUSTYLNICK, I. B., 1967, Astrofizika 3, 69.

RUCINSKI, S. M., 1969, Acta Astron. 19, 245.

RUCINSKI, S. M., 1970, Acta Astron. 20, 327.

SHOBBROOK, R. R., HERBISON-EVANS, D., JOHNSTON, I. D., and LOMB, N. R., 1969, Monthly Notices Roy. Astron. Soc. 145, 131

SMAK, J., 1970, Acta Astron. 20, 75.

SOBIESKI, S., 1965, Astrophys. J. Suppl. 12, 276.

STOTHERS, R., 1971, Nature 229, 180.

STOTHERS, R., and SIMON, N. R., 1969, Astrophys. J. 157, 673.

STRUVE, O., and BOBROVNIKOFF, N. T., 1925, Astrophys. J. 62, 139.

STRUVE, O., McNAMARA, D. H., KRAFT, R. P., KUNG, S. M., and WILliaMS, A. D., 1952, Astrophys. J. 116, 81.

STRUVE, O., McNAMARA, D. H., KUNG, S. M., and BEYMER, C., 1953, Astrophys. J. 118, 39.

STRUVE, O., MeNAMARA, D. H., and ZEBERGS, V., 1955, Astrophys. J. 122, 122.

STRUVE, O. SAHADE, J, HUANG, S.-S., and ZEBERGS, V., 1958, Astrophys. J. 128, 310.

WALKER, M. F., 1951, Publ. Astron. Soc. Pacific 63, 55.

WALKER, M. F., 1952, Astrophys. ]. 116, 106.

WALKER, M. F, 1954, Astrophys. J. 120, 58.

ZAHN, J. P., 1966a, Ann. Astrophys. 29, 313.

LAHN, J. P., 1966b, Ann. Astrophys. 29, 489.

ZAHN, J. P., 1966c, Ann. Astrophys. 29, 565.

VON ZEIPEL, H., 1924, Monthly Notices Roy. Astron. Soc. 84, 702.

\section{Discussion to the paper of LARSSON-LEANDER}

AIZENMAN: It would be of interest to begin a general photometric study of all early B stars. The justification for this has been demonstrated by the discovery by SHOBROOK of $\alpha$ Virginis as a $\beta$ Cephei variable. This star is the first confirmed $\beta$ Cephei variable to be found by photometric techniques. All of the others (18) were found by examination of their spectra.

Up till now everyone has believed that $\beta$ Cephei stars are intrinsically slow rotators with relatively sharp lines. But $\alpha$ Vir is a fast rotator $v \sin i \sim 160 \mathrm{~km} / \mathrm{sec}$ according to STRUVE) and has broad lines. It is clear that we have a distinct selection effect here, in the sense that it is easier to detect variability for sharp line stars rather than broad line stars. Such a selection effect can be avoided if we make photometric searches. If there are other $\beta$ Cephei stars which are rapid rotators we must reconsider the ideas of CLEMENT concerning these stars.

Now $\alpha$ Vir has been observed for almost 80 years and its discovery as a $\beta$ Cephei star allowed Dr. SMAK to examine observations made a long base-line and show that there was a secular decrease in the period of $\alpha$ Vir. This is the first $\beta$ Cephei star to show such an effect (over a long time) and before this one generally thought that $\beta$ Cephei periods showed secular increases (STRUVE 1955). This fact was one of the principal reasons why many people placed $\beta$ Cephei stars in the central hydrogen burning phase of evolution. Now Dr. SMAK is inclined to place $\alpha$ Vir in the general contraction phase because it shows a period decrease. It is clear that the evolutionary problem has now been reopened with the discovery of $\alpha$ Vir.

Photometric observations can be made by almost every reasonably equipped telescope. If an international effort were to be made to observe all of the early B stars (for a continuous period of about 6 hours for several nights), then we could hope to increase the number of $\beta$ Cephei stars. The resulting improved statistics might help us to understand these stars.

PERCY: Although some of Dr. HILL's stars have been confirmed to be variable, others have not. $I$ therefore urge observers to confirm the variability of these stars before further conclusions are drawn. 
HERCZEG : Has anybody ever observed an eclipse or part of an eclipse among $\beta \mathrm{CMa}$ stars? If the sample is comprising perhaps 100 stars as shown on the slide and if the periods are ranging from, say, 1-10 days, we have to expect dozens of the stars undergo eclipses.

LARSSON-LEANDER: No, as far as I know, no eclipses have been observed as yet.

MAVRIDIS: Do you think that the beat phenomena shown by TU Cas and similar cepheids with $\mathrm{P} \approx 2^{\mathrm{d}}$ could also be explained as the result of the presence of a close companion?

LARSSON-LEANDER: I cannot answer this question.

SMAK: The interferometric results now point quite clearly that $\alpha$ Vir $A$ is probably in the overall contraction phase following the core hydrogen burning. This is consistent with the period shortening. The data available thus seem to exclude the $\mu$-mechanism of STOTHERS and SIMON.

DETRE: The same conclusion that was mentioned by Dr. SMAK about $\beta$ CMa stars can be perhaps drawn also for dwarf Cepheids. Cerro Tololo observations of SX Phoenicis show that the radial velocity and light variations are not due to pulsation, they are probably caused by effects in a binary system.

PERCY: 1 . I do not believe that one can at present say that a given star as in the posthydrogen-exhaustion phase of evolution.

2. I shudder to think that $\beta \mathrm{CMa}$ stars may not pulsate!

3. I urge that observers try to establish whether any rapidly rotating early B stars are true $\beta \mathrm{CMa}$ stars.

LESH: I would like to make some remarks concerning the spectral classification of the $\beta$ Canis Majoris stars:

1. The "classical" $\beta$ Canis Majoris stars all have accurate MK types by MORGAN's group. They fall in the very narrow range $\mathrm{B} 0.5-\mathrm{B} 2$, III-IV (except for $\beta \mathrm{CMa}$ itself, which is luminosity class II - III.

2. Many of the stars found by HILL do not yet have accurate MK classifications. I am presently carrying out a program at the Meudon-Observatory to classify these stars. But at least one of them is an MK supergiant standard, and one of them is a B 3 V on the Scorpio-Centaurus main sequence. Therefore they do fall outside the narrow range defined by the classical $\beta$ CMa stars. This raises the question of whether HILL's stars should be called $\beta \mathrm{CMa}$ stars, even if their variability should be confirmed by subsequent photometric observations. In other words, is any variable $\mathrm{B}$ star a $\beta \mathrm{CMa}$ ? Or should a star be assigned in this class only if it obeys the period-luminosity relation, the phase relation between light and radial-velocity curves, etc., as defined by the classical $\beta$ CMa variables?

3. Finally, even using the best spectral classification, one finds that the $\beta \mathrm{CMa}$ stars fall in a region of the H-R diagram, where there are many normal, non-variable stars (as HILL found for the stars he observed). The separation of the variable and nonvariable stars in this region remains a challenging observational problem.

AIZENMAN: No HILL star has received confirmation by other observers. In fact, several have been already rejected. Thus the characteristics of the $\beta$ Cep stars as deduced from HILL's results should be regarded as highly dubious. For example, the question of whether the $\beta$ Cephei strip cuts the Main Sequence is of fundamental importance as far as the evolutionary state of these stars is concerned. Dr. SMAK has just said that $\alpha$ Virginis lies in the overall contraction phase following hydrogen exhaustion in the core. If a class V $\beta$ Cephei star were to be found this would pose some problems for evolution.

With respect to the $\delta$ Scuti stars, Mme. CHEVALIER in France has shown that the same mechanism that drives the Cepheid pulsations also works für $\delta$ Scuti stars. It is interesting to note that the star she found unstable was in the overall contraction phase. 